\title{
Pancreatic Kaposiform Hemangioendothelioma Not Responding to Sirolimus
}

\author{
Paloma Junco Triana ${ }^{1}$ Mariela Dore ${ }^{1}$ Vanesa Cerezo Nuñez ${ }^{1} \quad$ Javier Gomez Jimenez ${ }^{1}$ \\ Miriam Ferrero Miguel ${ }^{1}$ Mercedes González Díaz ${ }^{1}$ Joan Novo Ricardo ${ }^{2}$ Ane Andres ${ }^{1}$ \\ Manuel Lopez Santamaria ${ }^{1}$ Juan Carlos Lopez-Gutierrez ${ }^{3}$ \\ ${ }^{1}$ Department of Pediatric Surgery, La Paz Children's Hospital, Madrid, \\ Spain \\ 2 Department of Interventional Radiology, La Paz Children's Hospital, \\ Address for correspondence Paloma Junco Triana, Hospital \\ Universitario La Paz - Pediatric Surgery, Paseo de la Castellana 261, \\ Madrid, Spain \\ ${ }^{3}$ Division of Vascular Anomalies, Department of Pediatric Surgery, La \\ Paz Children's Hospital, Madrid, Spain \\ Eur J Pediatr Surg Rep 2017;5:e32-e35.
}

\begin{abstract}
Keywords

- kaposiform hemangioendothelioma

- pancreatic

- Sirolimus

Background Kaposiform hemangioendothelioma (KHE) is a vascular tumor frequently associated with Kasabach-Merritt phenomenon (KMP), characterized by severe thrombocytopenia and consumptive coagulopathy. Visceral involvement in $\mathrm{KHE}$ is rare. In our recent experience, sirolimus has shown to be an effective treatment in cutaneous KHE, becoming indeed the treatment of choice in KMP. We report a case of pancreatic KHE associated with KMP and refractory to sirolimus.

Case Report A 4-month-old infant is referred for obstructive jaundice $(10 \mathrm{mg} / \mathrm{dL}$ conjugated bilirubin) secondary to vascular pancreatic tumor. Magnetic resonance (MR) and immunohistochemistry were compatible with KHE, but the tumor was considered unresectable. We initiated sirolimus $\left(0.8 \mathrm{mg} / \mathrm{m}^{2} / 12 \mathrm{~h}\right)$ to treat KMP, and interventional radiology was performed for percutaneous biliary diversion. This procedure prompted KMP (platelets: $51,000 / \mu \mathrm{L}$ ). Sirolimus treatment for 7 days showed no effect; therefore, we started our VAT protocol (vincristine/aspirine/ticlopi$\mathrm{din})$ with great response after 10 days (platelets: 3,70,000/ $\mu \mathrm{L}$ ). Three months later, percutaneous biliary diversion was replaced by a biliary stent. The tumor disappeared leaving fibrosis and dilatation of biliary tract needing hepaticojejunostomy 6 months later.

Discussion It is difficult to establish protocols for an unusual presentation of a tumor with different targets. This is a reason collaborative multicenter studies should be performed. Management of obstructive jaundice secondary to a tumor that usually regresses in 10 years is an added challenge; therefore, the management should be led by a multidisciplinary team.

Sirolimus treatment in cutaneous KHE has been described as successful in the literature, as well as in our own experience; however, it failed in our first patient with visceral KHE. We need to investigate the different response to pharmacological agents in tumors with similar histopathology, but with visceral involvement.
\end{abstract}

received

April 18, 2017

accepted after revision

June 18, 2017
DOI https://doi.org/

$10.1055 / \mathrm{s}-0037-1604358$.

ISSN 2194-7619. (c) 2017 Georg Thieme Verlag KG

Stuttgart · New York
License terms

(1) (1) 


\section{New Insights and the Importance for the Pediatric Surgeon}

Kaposiform hemangioendothelioma (KHE) with pancreatic involvement is rare, and it suggests different behavior than the cutaneous involvement. Even histopathology is similar in both anatomical locations, resistance to mammalian target of rapamycin (mTOR) inhibitors treatment in pancreatic involvement suggest the possibility of a different molecular profile.

\section{Introduction}

Kaposiform hemangioendothelioma (KHE) and tufted angioma (TA) are vascular tumors with clinical and histopathological overlap in the same disease, TA being at the less aggressive part of the spectrum. KHE is an infiltrative vascular tumor of endothelial and lymphatic cell lineage that usually presents in childhood, predominantly below 1 year of age. ${ }^{1}$ KHE can appear in skin, deep soft tissue, retroperitoneum, and mediastinum, and it rarely takes visceral involvement. KHE is frequently associated with Kasabach-Merrit phenomenon (KMP), characterized by severe thrombocytopenia and consumptive coagulopathy, especially in large tumors $(>5 \mathrm{~cm})$.

Mammalian target of rapamycin (mTOR) inhibitors, particularly sirolimus, have been shown to be an effective treatment in cutaneous KHE, becoming indeed the treatment of choice in $\mathrm{KMP}^{2}$ From $<40$ retroperitoneal KHE reported in English literature, only two involved pancreas, one was treated with Whipple operation, and the other responded to sirolimus.,

We report a case of pancreatic KHE associated with KMP refractory to sirolimus.

\section{Case Report}

A 4-month-old infant is referred to our hospital for obstructive jaundice secondary to a vascular pancreatic tumor. Her history revealed the presence of vomiting, acholic stools, and progressive jaundice for 5 weeks of life. Physical examination showed cutaneous and conjunctival jaundice and minor hepatomegaly. Laboratory testing disclosed elevated liver enzymes (glutamic oxaloacetic transaminase [GOT] $178 \mathrm{IU} / \mathrm{L}$, glutamic-pyruvic transaminase [GPT] $128 \mathrm{IU} / \mathrm{L}$, and gamma-glutamyl transferase [GGT] $608 \mathrm{IU} / \mathrm{L})$, elevated conjugated bilirubin $(10 \mathrm{mg} / \mathrm{dL})$, and a minor decrease in platelets $(109,000 / \mu \mathrm{L})$. Abdominal ultrasound (US) and MR showed a vascular mass at the pancreatic head $(3.3 \times 2.4 \times 1.8 \mathrm{~cm})$, a distended gallbladder and a dilated biliary tract (up to $7.2 \mathrm{~mm}$ ) ( - Fig. 1). An open laparotomy for biopsy was performed and immunohistochemistry profile was compatible with KHE (-Fig. 2).

She was admitted to our hospital, and, after considering the tumor unresectable, sirolimus was started at the initial dose of $0.8 \mathrm{mg} / \mathrm{m}^{2} / 12 \mathrm{~h}$ to treat KHE and to prevent KMP. To resolve obstructive jaundice, interventional radiology was performed for percutaneous biliary diversion. This procedure prompted KMP with thrombocytopenia (platelets $51,000 / \mu \mathrm{L}$ ), consumptive coagulopathy (prothrombin activity 45\%), and microangiopathic anemia. Percutaneous biliary diversion needed relocation 6 days after the placement due to obstruction, and after manipulation, the patient developed duodenal hematoma, which resolved with drainage. Sirolimus treatment for 7 days showed no improvement; therefore, we started our VAT protocol (vincristine/aspirine/ticlopidin) after placing a central catheter. Platelets showed great response after 10 days $(3,70,000 / \mu \mathrm{L})$.

Three months later, vincristine was withdrawn in light of platelets stabilization, and percutaneous biliary diversion was replaced by a biodegradable biliary stent (diameter $7 \mathrm{~mm}$ and length $36 \mathrm{~mm}$ ) due to persistence of biliary tract dilation. The patient presented self-limited hematemesis and hematochezia after stent implant.
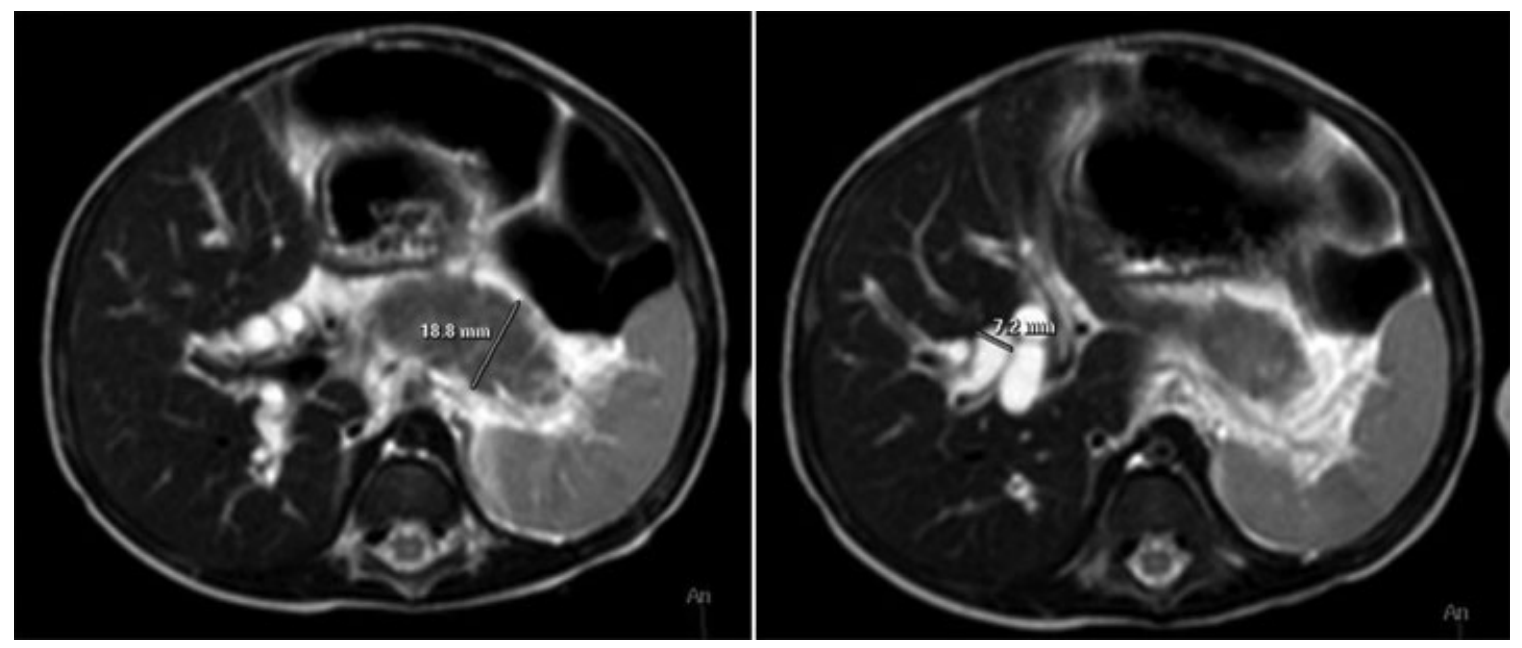

Fig. 1 Magnetic resonance findings vascular tumor at the pancreatic head $(3.3 \times 2.4 \times 1.8 \mathrm{~cm})$ and a dilated biliary tract $(7.2 \mathrm{~mm})$. 

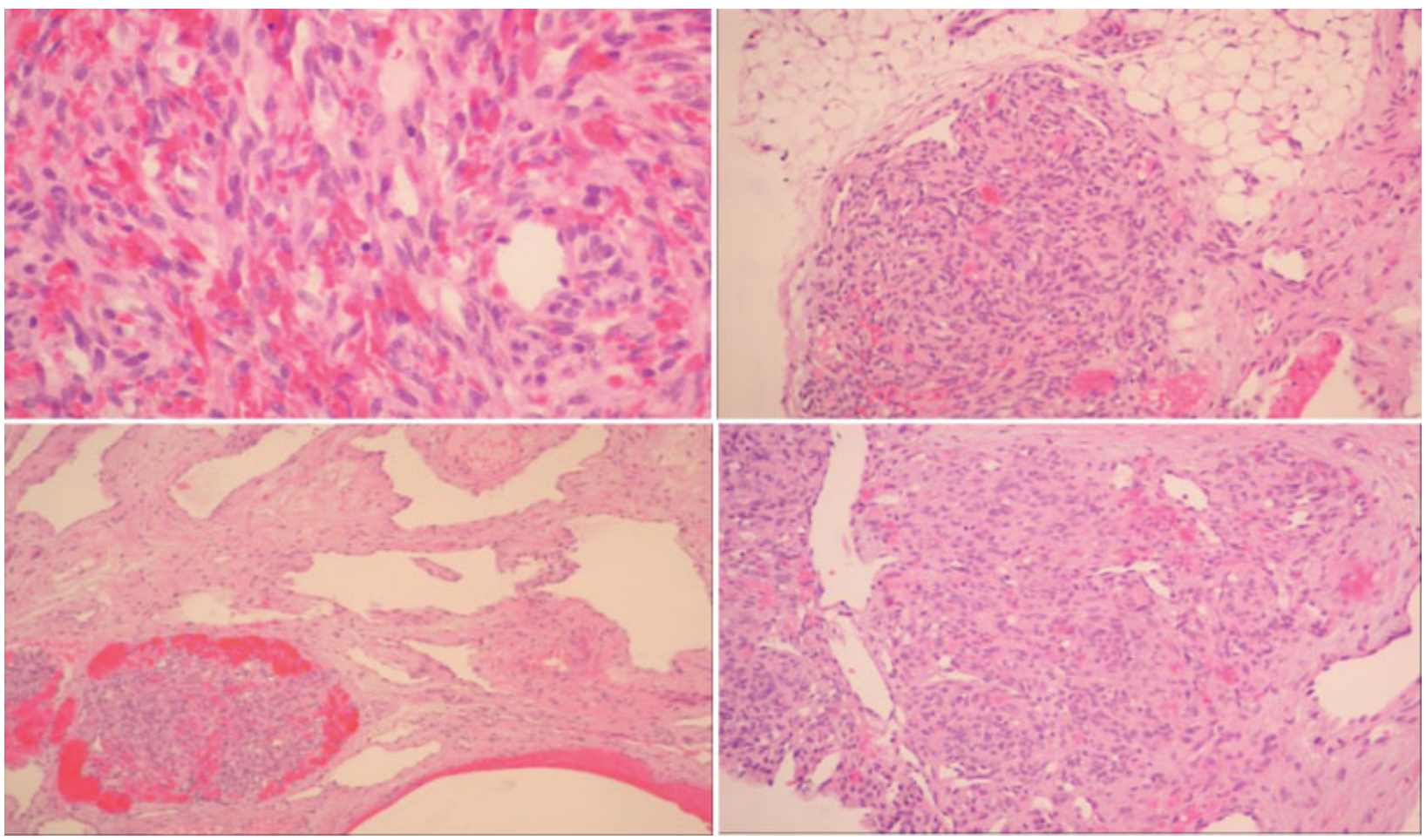

Fig. 2 Pathology findings pancreatic kaposiform hemangioendothelioma. Hematoxylin-eosin staining shows abnormal proliferation of spindle endothelial cells in pancreatic tissue and slit-like lumen formations.

Three months later, we performed a control MR revealing tumor involution leaving fibrosis. Abdominal US showed dilation of biliary tract persistence (up to $8-9 \mathrm{~mm}$ ); therefore, open hepaticojejunostomy with Roux-en-Y reconstruction was performed, and the stent was removed. The patient was discharged 7 days after surgery.

The patient is asymptomatic and on oral feeds at present.

\section{Discussion}

KHE typically presents during childhood as a vascular tumor, affecting trunk, extremities, and retroperitoneum. ${ }^{1}$ It rarely presents with visceral involvement, with only a few dozen cases published in the literature and only two cases with pancreatic settlement known.,4 KMP is a frequent complication, especially in large tumors and visceral involvement, in which platelet trapping within the tumor can lead to life-threatening thrombocytopenia. Although our patient presented a tumor $<5 \mathrm{~cm}$, retroperitoneal and visceral involvement have shown an increased risk of KMP. ${ }^{1}$ KHE is a tumor that shrinks in time and usually regresses in 10 years, but it may leave a dense fibrosis behind. This tendency to disappear makes it easier to decide close follow-up without treatment, unless arising of KMP or other complications, such as obstructive jaundice, is observed in our patient.

Multiple medical therapies have been used in the treatment of KHE and KMP, including systemic steroids, vincristine, interferon-alfa, and aspirin. ${ }^{5}$ Our stablished protocol for KMP involves a combination of chemotherapy with vincristine $(0.05 \mathrm{mg} / \mathrm{kg}$ given weekly) and double antiaggregation with ticlopidine $(10 \mathrm{mg} / \mathrm{kg} /$ day $)$ and aspirin $(10 \mathrm{mg} / \mathrm{kg} / \mathrm{day}) .{ }^{6,7}$ Sirolimus treatment in cutaneous KHE has been described as successful in the literature, as well as in our own experience; however, it failed in our patient with visceral KHE. ${ }^{8,9}$ Side effects of medical therapy and recurrence rates of KHE are not described well; therefore, long-term follow-up is mandatory in these patients.

\section{Conclusion}

KHE remains the most aggressive of benign vascular tumors. Pancreatic involvement is exceedingly rare, and data from an eventual different behavior in comparison with the cutaneous involvement are not available. Even considering that KHE histopathology is similar in both anatomical locations, resistance to $\mathrm{mTOR}$ inhibitors treatment suggests the possibility of a different molecular profile. VAT protocol remains a safe and successful pharmacological therapy in the management of KMP.

\section{Conflict of Interest}

None.

\section{References}

1 Croteau SE, Liang MG, Kozakewich HP, et al. Kaposiform hemangioendothelioma: atypical features and risks of Kasabach-Merritt phenomenon in 107 referrals. J Pediatr 2013;162(01):142-147 
2 Ji Y, Chen S, Xiang B, et al. Sirolimus for the treatment of progressive kaposiform hemangioendothelioma: a multicenter retrospective study. Int J Cancer 2017;141(04):848-855

3 Leung M, Chao NS, Tang PM, Liu K, Chung KL. Pancreatic kaposiform hemangioendothelioma presenting with duodenal obstruction and Kasabach-Merritt phenomenon: a neonate cured by Wipple operation. European J Pediatr Surg Rep 2014;2(01):7-9

4 Wang C, Li Y, Xiang B, et al. Successful management of pancreatic kaposiform hemangioendothelioma with sirolimus: case report and literature review. Pancreas 2017;46(05):e39-e41

5 Drolet BA, Trenor CC III, Brandão LR, et al. Consensus-derived practice standards plan for complicated kaposiform hemangioendothelioma. J Pediatr 2013;163(01):285-291
6 Tlougan BE, Lee MT, Drolet BA, Frieden IJ, Adams DM, Garzon MC. Medical management of tumors associated with Kasabach-Merritt phenomenon: an expert survey. J Pediatr Hematol Oncol 2013;35(08):618-622

7 Fernandez-Pineda I, Lopez-Gutierrez JC, Ramirez G, Marquez C. Vincristine-ticlopidine-aspirin: an effective therapy in children with Kasabach-Merritt phenomenon associated with vascular tumors. Pediatr Hematol Oncol 2010;27(08):641-645

8 Triana P, Dore M, Cerezo VN, et al. Sirolimus in the treatment of vascular anomalies. Eur J Pediatr Surg 2017;27(01):86-90

9 Hammill AM, Wentzel M, Gupta A, et al. Sirolimus for the treatment of complicated vascular anomalies in children. Pediatr Blood Cancer 2011;57(06):1018-1024 